Proyecciones Journal of Mathematics

Vol. 41, No 1, pp. 335-351, February 2022.

Universidad Católica del Norte

Antofagasta - Chile

\title{
An extension of biconservative timelike hypersurfaces in Einstein space
}

\author{
Firooz Pashaie \\ University of Maragheh, Iran \\ Received : June 2021. Accepted : September 2021
}

\begin{abstract}
$A$ well-known conjecture of Bang-Yen Chen says that the only biharmonic Euclidean submanifolds are minimal ones, which affirmed by himself for surfaces in 3-dimensional Euclidean space, $E^{3}$. We consider an extended version of Chen conjecture (namely, $L_{k}$-conjecture) on Lorentzian hypersurfaces of the pseudo-Euclidean space $E_{1}^{4}$ (i.e. the Einstein space). The biconservative submanifolds in the Euclidean spaces are submanifolds with conservative stress-energy with respect to the bienergy functional. In this paper, we consider an extended condition (namely, $L_{k}$-biconservativity) on non-degenerate timelike hypersurfaces of the Einstein space $E_{1}^{4}$. A Lorentzian hypersurface $x: M_{1}^{3} \rightarrow E_{1}^{4}$ is called $L_{k}$-biconservative if the tangent part of $L_{k}^{2} x$ vanishes identically. We show that $L_{k}$-biconservativity of a timelike hypersurface of $E_{1}^{4}$ (with constant kth mean curvature and some additional conditions) implies that its $(k+1)$ th mean curvature is constant.
\end{abstract}

Keywords: Timelike hypersurface, Biconservative, $L_{k}$-biconservative.

2010 Mathematics Subject Classification: Primary: 53-02, 53C40, 53C42; Secondary 58G25. 


\section{Introduction}

The main geometric motivation of the subject of biconservative hypersurfaces is a well-known conjecture of Bang-Yen Chen (in 1987) which states that each biharmonic surface in Euclidean 3 -spaces $E^{3}$ is harmonic. In 1992, Dimitrić proved that any biharmonic hypersurface in $E^{m}$ with at most two distinct principal curvatures is minimal ([9]). Let $\phi: M^{n} \rightarrow E^{n+1}$ denotes an isometric immersion of a hypersurface $M^{n}$ into the $(n+1)$-dimensional Euclidean space with the Laplace operator $\Delta$, the shape operator $S$ associated to a unit normal vector field $\mathbf{n}$ and the ordinary mean curvature $H$ on $M^{n}$. The hypersurface $M^{n}$ is said to be harmonic if $\phi$ satisfies condition $\Delta \phi=0$. It is said to be biharmonic if $\phi$ satisfies condition $\Delta^{2} \phi=0$. Also, $M^{n}$ is said to be biconservative if the tangential part of $\Delta^{2} \phi$ vanishes identically. A famous law due to Beltrami says that $\Delta \phi=-n H \mathbf{n}$, so the condition $\Delta \phi=0$ is equivalent to $H \equiv 0$ and the condition $\Delta^{2} \phi=0$ is equivalent to $\Delta(H \mathbf{n})=0$. In 1995, Hasanis and Vlachos proved an extension of Chen's result to the hypersurfaces in Euclidean 4-space ([10]). Also, in 2013, Akutagawa and Maeta ([1]) have generalized Chen's conjecture on submanifolds in Euclidean $n$-space. As an extended case, a hypersurface $x: M_{p}^{3} \rightarrow E_{s}^{4}$, whose mean curvature vector field is an eigenvector of the Laplace operator $\Delta$, has been studied, for instance, in $[7,8]$ for the Euclidean case (where, $p=s=0$ ), and for the Lorentz case in [3, 4] (when $s=1$ and $p=0,1$ ). On the other hand, Chen himself had found a good relation between the finite type hypersurfaces and biharmonic ones. The theory of finite type hypersurfaces is a well-known subject interested by Chen (for instance, in $[5,6]$ ) and also L. J. Alias, S.M.B. Kashani and others. In [11], Kashani has studied the notion of $L_{1}$-finite type Euclidean hypersurfaces as an extension of finite type ones. One can see main results in Chapter 11 of Chen's book ([5]).

The map $L_{1}$ is an extension of the Laplace operator $L_{0}=\Delta$, which stands for the linearized operator of the first variation of the 2 th mean curvature of the hypersurface (see, for instance, $[2,12,16,17,19]$ ). This operator is defined by $L_{1}(f)=\operatorname{tr}\left(P_{1} \circ \nabla^{2} f\right)$ for any $f \in C^{\infty}(M)$, where $P_{1}=n H I-S$ denotes the first Newton transformation associated to the second fundamental from of the hypersurface and $\nabla^{2} f$ is the hessian of $f$. It is interesting to generalize the definition of biharmonic hypersurface by replacing $\Delta$ by $L_{1}$. Recently, in [14], we have studied the $L_{1^{-}}$ biharmonic spacelike hypersurfaces in 4-dimentional Minkowski space $E_{1}^{4}$. In this paper, we study the $L_{k}$-biconservative Lorentzian hypersurfaces in 
the Einstein space $E_{1}^{4}$. We show that, every $L_{k}$-biconservative Lorentzian hypersurface $x: M_{1}^{3} \rightarrow E_{1}^{4}$, with constant $k$ th mean curvature and some additional conditions on principal curvatures, has constant $(k+1)$ th mean curvature.

\section{Preliminaries}

In this section, we restate some preliminaries from $[2,12,13]$ and [15][18]. The 4-dimensional Minkowski space, denoted by $E_{1}^{4}$, is the real vector space $R^{4}$ equipped with the scalar product $\langle x, y\rangle:=-x_{1} y_{1}+\sum_{i=2}^{4} x_{i} y_{i}$, for every $x, y \in R^{4}$. Any nondegenerate hypersurface $M_{p}^{3}$ of $E_{1}^{4}$, can be endowed with a Riemannian or Lorentzian induced metric of index $p=0$ or $p=1$, respectively. Our study will be on a Lorentzian hypersurface of $E_{1}^{4}$, denoted by an isometric immersion $x: M_{1}^{3} \rightarrow E_{1}^{4}$. The symbols $\tilde{\nabla}$ and $\bar{\nabla}$ stand for the Levi-Civita connection on $M_{1}^{3}$ and $E_{1}^{4}$, respectively. For every tangent vector fields $X$ and $Y$ on $M$, the Gauss formula is given by $\bar{\nabla}_{X} Y=\tilde{\nabla}_{X} Y+\langle S X, Y>\mathbf{n}$, for every $X, Y \in \chi(M)$, where, $\mathbf{n}$ is a (locally) unit normal vector field on $M$ and $S$ is the shape operator of $M$ relative to $\mathbf{n}$. Every non null vector $X \in E_{1}^{4}$ is called time-like, light-like or space-like if $\langle X, X\rangle$ is negative, zero or positive, respectively.

Definition 2.1. For a Lorentzian vector space $V_{1}^{3}$, a basis $\mathcal{B}:=\left\{e_{1}, e_{2}, e_{3}\right\}$ is said to be orthonormal if it satisfies $\left\langle e_{i}, e_{j}\right\rangle=\epsilon_{i} \delta_{i}^{j}$ for $i, j=1,2,3$, where $\epsilon_{1}=-1$ and $\epsilon_{i}=1$ for $i=2,3$. As usual, $\delta_{i}^{j}$ stands for the Kronecker function. $\mathcal{B}$ is called pseudo - orthonormal if it satisfies $\left\langle e_{1}, e_{1}\right\rangle=<$ $e_{2}, e_{2}>=0,<e_{1}, e_{2}>=-1$ and $\left\langle e_{i}, e_{j}>=\delta_{i}^{j}\right.$, for $i=1,2,3$ and $j=3$.

As well-known, the shape operator of the Lorentzian hypersurface $M_{1}^{3}$, as a self-adjoint linear map on the tangent space of $M_{1}^{3}$, can be put into one of four possible canonical matrix forms, usually denoted by $I, I I, I I I$ and $I V$. Where, in cases $I$ and $I V$, with respect to an orthonormal basis of the tangent space of $M_{1}^{3}$, the matrix representation of the induced metric on $M_{1}^{3}$ is

$$
G_{1}=\left(\begin{array}{ccc}
-1 & 0 & 0 \\
0 & 1 & 0 \\
0 & 0 & 1
\end{array}\right)
$$


and the shape operator $S$ of $M_{1}^{3}$ can be put into matrix forms

$$
B_{1}=\left(\begin{array}{ccc}
\lambda_{1} & 0 & 0 \\
0 & \lambda_{2} & 0 \\
0 & 0 & \lambda_{3}
\end{array}\right) \quad \text { and } \quad B_{4}=\left(\begin{array}{ccc}
\kappa & \lambda & 0 \\
-\lambda & \kappa & 0 \\
0 & 0 & \eta
\end{array}\right), \quad(\lambda \neq 0)
$$

respectively. For cases $I I$ and $I I I$, using a pseudo-orthonormal basis of the tangent space of $M_{1}^{3}$, the induced metric on $M_{1}^{3}$ has matrix form

$$
G_{2}=\left(\begin{array}{ccc}
0 & 1 & 0 \\
1 & 0 & 0 \\
0 & 0 & 1
\end{array}\right)
$$

and the shape operator $S$ of $M_{1}^{3}$ can be put into matrix forms

$$
B_{2}=\left(\begin{array}{ccc}
\kappa & 0 & 0 \\
1 & \kappa & 0 \\
0 & 0 & \lambda
\end{array}\right) \quad \text { and } \quad B_{3}=\left(\begin{array}{ccc}
\kappa & 0 & 0 \\
0 & \kappa & 1 \\
-1 & 0 & \kappa
\end{array}\right)
$$

respectively. In case $I V$, the matrix $B_{4}$ has two conjugate complex eigenvalues $\kappa \pm i \lambda$, but in other cases the eigenvalues of the shape operator are real numbers.

Remark 2.2. In two cases $I I$ and $I I I$, one can substitute the pseudoorthonormal basis $\mathcal{B}:=\left\{e_{1}, e_{2}, e_{3}\right\}$ by orthonormal basis $\tilde{\mathcal{B}}:=\left\{\tilde{e_{1}}, \tilde{e_{2}}, e_{3}\right\}$ where $\tilde{e_{1}}:=\frac{1}{2}\left(e_{1}+e_{2}\right)$ and $\tilde{e_{2}}:=\frac{1}{2}\left(e_{1}-e_{2}\right)$. Therefore, we obtain new matrix representations $\tilde{B}_{2}$ and $\tilde{B}_{3}$ (instead of $B_{2}$ and $B_{3}$, respectively) as $\tilde{B}_{2}=\left(\begin{array}{ccc}\kappa+\frac{1}{2} & \frac{1}{2} & 0 \\ -\frac{1}{2} & \kappa-\frac{1}{2} & 0 \\ 0 & 0 & \lambda\end{array}\right) \quad$ and $\quad \tilde{B}_{3}=\left(\begin{array}{ccc}\kappa & 0 & \frac{\sqrt{2}}{2} \\ 0 & \kappa & -\sqrt{2} / 2 \\ -\frac{\sqrt{2}}{2} & -\frac{\sqrt{2}}{2} & \kappa\end{array}\right)$

After this changes, to unify the notations we denote the orthonormal basis by $\mathcal{B}$ in all cases. 
Notation: According to four possible matrix representations of the shape operator of $M_{1}^{3}$, we define its principal curvatures, denoted by unified notations $\kappa_{i}$ for $i=1,2,3$, as follow.

In case $I$, we put $\kappa_{i}:=\lambda_{i}$, for $i=1,2,3$, where $\lambda_{i}$ 's are the eigenvalues of $B_{1}$.

In cases $I I$, where the matrix representation of $S$ is $\tilde{B}_{2}$, we take $\kappa_{i}:=\kappa$ for $i=1,2$, and $\kappa_{3}:=\lambda$.

In case $I I I$, where the shape operator has matrix representation $\tilde{B}_{3}$, we take $\kappa_{i}:=\kappa$ for $i=1,2,3$.

Finally, in the case $I V$, where the shape operator has matrix representation $\tilde{B}_{4}$, we put $\kappa_{1}=\kappa+i \lambda, \kappa_{2}=\kappa-i \lambda$, and $\kappa_{3}:=\eta$.

The characteristic polynomial of $S$ on $M_{1}^{3}$ is of the form

$$
Q(t)=\prod_{i=1}^{3}\left(t-\kappa_{i}\right)=\sum_{j=0}^{3}(-1)^{j} s_{j} t^{3-j},
$$

where, $s_{0}:=1, s_{1}=\sum_{j=1}^{3} \kappa_{j}, s_{2}:=\sum_{1 \leq i_{1}<i_{2} \leq 3} \kappa_{i_{1}} \kappa_{i_{2}}$ and $s_{3}:=\kappa_{1} \kappa_{2} \kappa_{3}$.

For $k=1,2,3$, the $k$ th mean curvature $H_{k}$ of $M$ is defined by $H_{k}=$ $\frac{1}{\left(\begin{array}{l}3 \\ k\end{array}\right)} s_{k}$. When $H_{k}$ is identically null, $M_{1}^{n}$ is said to be $(k-1)$-minimal.

Definition 2.3. (i) A timelike hypersurface $x: M_{1}^{3} \rightarrow E_{1}^{4}$, with diagonalizable shape operator, is said to be isoparametric if all of it's principal curvatures are constant.

(ii) A timelike hypersurface $x: M_{1}^{3} \rightarrow E_{1}^{4}$, with non-diagonalizable shape operator, is said to be isoparametric if the minimal polynomial of the shape operator is constant.

Remark 2.4. Here we remember Theorem 4.10 from [13], which assures us that there is no isoparametric timelike hypersurface of $E_{1}^{4}$ with complex principal curvatures.

The Newton transformations on the hypersurface, $P_{k}: \chi(M) \rightarrow \chi(M)$, is defined by

$$
P_{0}=I, \quad P_{k}=s_{k} I-S \circ P_{k-1}, \quad(k=1,2,3),
$$

where, $I$ is the identity map. The explicit formula $P_{k}=\sum_{i=0}^{k}(-1)^{i} s_{k-i} S^{i}$ (where $S^{0}=I$ ) gives that, $P_{k}$ is self-adjoint and it commutes with $S$ (see $[2,16])$. 
Now, we define a notation as

$(2.2) \mu_{j ; k}=\sum_{l=0}^{k}(-1)^{l}\left(\begin{array}{l}n \\ k-l\end{array}\right) H_{k-l} \kappa_{j}^{l} . \quad(1 \leq j \leq 3,1 \leq k<3)$

Corresponding to the four possible forms $\tilde{B}_{i}$ (for $1 \leq i \leq 4$ ) of $S$, the Newton transformation $P_{j}$ has different representations. In the case $I$, where $S_{p}=\tilde{B}_{1}$, we have $P_{j}(p)=\operatorname{diag}\left[\mu_{1 ; j}(p), \mu_{2 ; j}(p), \mu_{3 ; j}(p)\right]$, for $j=1,2$.

When $S=B_{2}$ (in the case $I I$ ), we have

$P_{1}=\left(\begin{array}{ccc}\kappa+\lambda-\frac{1}{2} & -\frac{1}{2} & 0 \\ \frac{1}{2} & \kappa+\lambda+\frac{1}{2} & 0 \\ 0 & 0 & 2 \kappa\end{array}\right), \quad P_{2}=\left(\begin{array}{ccc}\left(\kappa-\frac{1}{2}\right) \lambda & -\frac{1}{2} \lambda & 0 \\ \frac{1}{2} \lambda & \left(\kappa+\frac{1}{2}\right) \lambda & 0 \\ 0 & 0 & \kappa^{2}\end{array}\right)$.

In the case $I I I$, we have $S_{p}=B_{3}$, and

$$
P_{1}=\left(\begin{array}{ccc}
2 \kappa & 0 & -\frac{\sqrt{2}}{\sqrt{2}} \\
0 & 2 \kappa & \frac{\sqrt{2}}{2} \\
\frac{\sqrt{2}}{2} & \frac{\sqrt{2}}{2} & 2 \kappa
\end{array}\right), \quad P_{2}=\left(\begin{array}{ccc}
\kappa^{2}-\frac{1}{2} & -\frac{1}{2} & -\frac{\sqrt{2}}{2} \kappa \\
\frac{1}{2} & \kappa^{2}+\frac{1}{2} & \frac{\sqrt{2}}{2} \kappa \\
\frac{\sqrt{2}}{2} \kappa & \frac{\sqrt{2}}{2} \kappa & \kappa^{2}
\end{array}\right)
$$

In the case $I V, S=B_{4}$,

$$
P_{1}=\left(\begin{array}{ccc}
\kappa+\eta & -\lambda & 0 \\
\lambda & \kappa+\eta & 0 \\
0 & 0 & 2 \kappa
\end{array}\right), \quad P_{2}=\left(\begin{array}{ccc}
\kappa \eta & -\lambda \eta & 0 \\
\lambda \eta & \kappa \eta & 0 \\
0 & 0 & \kappa^{2}+\lambda^{2}
\end{array}\right) .
$$

Fortunately, in all cases we have the following important identities, similar to those in $[2,16]$.

$$
\operatorname{tr}\left(P_{1}\right)=6 H_{1}, \operatorname{tr}\left(P_{2}\right)=3 H_{2}, \operatorname{tr}\left(P_{1} \circ S\right)=6 H_{2}, \operatorname{tr}\left(P_{2} \circ S\right)=3 H_{3},
$$

(2.4) $\operatorname{tr} S^{2}=9 H_{1}^{2}-6 H_{2}, \operatorname{tr}\left(P_{1} \circ S^{2}\right)=9 H_{1} H_{2}-3 H_{3}, \operatorname{tr}\left(P_{2} \circ S^{2}\right)=3 H_{1} H_{3}$.

The linearized operator arisen from the first variation of the $(j+1)$ th mean curvature of $M$ denoted by $L_{j}: \mathcal{C}^{\infty}(M) \rightarrow \mathcal{C}^{\infty}(M)$ is defined by the formula $L_{j}(f):=\operatorname{tr}\left(P_{j} \circ \nabla^{2} f\right)$, where, $\left\langle\nabla^{2} f(X), Y\right\rangle=\left\langle\nabla_{X} \nabla f, Y>\right.$ for every $X, Y \in \chi(M)$. Associated to the orthonormal frame $\left\{e_{1}, e_{2}, e_{3}\right\}$ of 
tangent space on a local coordinate system in the hypersurface $x: M_{1}^{3} \rightarrow$ $E_{1}^{4}, L_{j}(f)$ has an explicit expression as

$$
L_{j}(f)=\sum_{i=1}^{3} \epsilon_{i} \mu_{i, j}\left(e_{i} e_{i} f-\nabla_{e_{i}} e_{i} f\right) .
$$

For a Lorentzian hypersurface $x: M_{1}^{3} \rightarrow E_{1}^{4}$, with a chosen (local) unit normal vector field $\mathbf{n}$, for an arbitrary vector $a \in E_{1}^{4}$ we use the decomposition $a=a^{T}+a^{N}$ where $a^{T} \in T M$ is the tangential component of $a, a^{N} \perp T M$, and we have the following formulae from $[2,16]$.

$$
\begin{gathered}
\nabla<x, a>=a^{T}, \nabla<\mathbf{n}, a>=-S a^{T}, L_{1} x=6 H_{2} \mathbf{n}, L_{2} x=3 H_{3} \mathbf{n} \\
L_{1} \mathbf{n}=-3 \nabla H_{2}-3\left[3 H_{1} H_{2}-H_{3}\right] \mathbf{n}, \\
L_{2} \mathbf{n}=-\nabla H_{3}-\left[3 H_{1} H_{3}\right] \mathbf{n}, \\
L_{1}^{2} x=6\left[2 P_{2} \nabla H_{2}-9 H_{2} \nabla H_{2}\right]+6\left[L_{1} H_{2}-9 H_{1} H_{2}^{2}+3 H_{2} H_{3}\right] \mathbf{n}, \\
L_{2}^{2} x=-9 H_{3} \nabla H_{3}+3\left(L_{2} H_{3}-3 H_{1} H_{3}^{2}\right) \mathbf{n} .
\end{gathered}
$$

Assume that a hypersurface $x: M_{1}^{3} \rightarrow E_{1}^{4}$ satisfies the condition $L_{k}^{2} x=$ 0 for an integer $k \in\{0,1,2\}$, then it is said to be $L_{k}$-biharmonic. In the case $k=0$, we have $L_{0}=\Delta$ and $L_{0}$-biharmoniciy is the same ordinary harmonicity which has been studied in $[3,4]$. By equalities $(2.8)$, a hypersurface $x: M_{1}^{3} \rightarrow E_{1}^{4}$ is $L_{1}$-biharmonic if and only if it satisfies two following conditions:

$$
\begin{aligned}
& \text { (i) } L_{1} H_{2}=3\left(3 H_{1} H_{2}^{2}-H_{2} H_{3}\right)=H_{2} \operatorname{tr}\left(S^{2} \circ P_{1}\right), \\
& \text { (ii) } P_{2} \nabla H_{2}=\frac{9}{2} H_{2} \nabla H_{2} .
\end{aligned}
$$

A timelike hypersurface $x: M_{1}^{3} \rightarrow E_{1}^{4}$ is said to be $L_{1}$-biconservative, if its 2nd mean curvature satisfies the condition (2.9)(ii).

Also, $x: M_{1}^{3} \rightarrow E_{1}^{4}$ is $L_{2}$-biharmonic if and only if it satisfies two following conditions:

$$
\text { (i) } L_{2} H_{3}=3 H_{1} H_{3}^{2}=H_{3} \operatorname{tr}\left(S^{2} \circ P_{2}\right), \quad \text { (ii) } H_{3} \nabla H_{3}=0 .
$$

A timelike hypersurface $x: M_{1}^{3} \rightarrow E_{1}^{4}$ is said to be $L_{2}$-biconservative, if its 3rd mean curvature satisfies the condition (2.10)(ii). 
The structure equations of $E_{1}^{4}$ are given by

$$
\begin{gathered}
d \omega_{i}=\sum_{j=1}^{4} \omega_{i j} \wedge \omega_{j}, \quad \omega_{i j}+\omega_{j i}=0, \\
d \omega_{i j}=\sum_{l=1}^{4} \omega_{i l} \wedge \omega_{l j} .
\end{gathered}
$$

With restriction to $M$, we have $\omega_{4}=0$ and then,

$$
0=d \omega_{4}=\sum_{i=1}^{3} \omega_{4, i} \wedge \omega_{i}
$$

By Cartan's lemma, there exist functions $h_{i j}$ such that

$$
\omega_{4, i}=\sum_{j=1}^{3} h_{i j} \omega_{j}, \quad h_{i j}=h_{j i} .
$$

This gives the second fundamental form of $M$, as $B=\sum_{i, j} h_{i j} \omega_{i} \omega_{j} e_{4}$. The mean curvature $H$ is given by $H=\frac{1}{3} \sum_{i=1}^{3} h_{i i}$. From (2.11)-(2.14) we obtain the structure equations of $M$ as follow.

$$
\begin{gathered}
d \omega_{i}=\sum_{j=1}^{3} \omega_{i j} \wedge \omega_{j}, \omega_{i j}+\omega_{j i}=0, \\
d \omega_{i j}=\sum_{k=1}^{3} \omega_{i k} \wedge \omega_{k j}-\frac{1}{2} \sum_{k, l=1}^{3} R_{i j k l} \omega_{k} \wedge \omega_{l},
\end{gathered}
$$

for $i, j=1,2,3$, and the Gauss equations

$$
R_{i j k l}=\left(h_{i k} h_{j l}-h_{i l} h_{j k}\right),
$$

where $R_{i j k l}$ denotes the components of the Riemannian curvature tensor of $M$.

Let $h_{i j k}$ denote the covariant derivative of $h_{i j}$.

We have

$$
d h_{i j}=\sum_{k=1}^{3} h_{i j k} \omega_{k}+\sum_{k=1}^{3} h_{k j} \omega_{i k}+\sum_{k=1}^{3} h_{i k} \omega_{j k} .
$$


Thus, by exterior differentiation of (2.14), we obtain the Codazzi equation

$$
h_{i j k}=h_{i k j} .
$$

Now we recall the definition of an $L_{k}$-finite type hypersurface from [11], which is the basic notion of the paper.

Definition 2.5. An isometrically immersed hypersurface $x: M_{1}^{3} \rightarrow E_{1}^{4}$ is said to be of $L_{k}$-finite type if $x$ has a finite decomposition $x=\sum_{i=0}^{m} x_{i}$, for some positive integer $m$, satisfying the condition $L_{k} x_{i}=\beta_{i} x_{i}$, where, $\beta_{i} \in R$ and $x_{i}: M^{3} \rightarrow E_{1}^{4}$ is smooth maps, for $i=1,2, \cdots, m$, and $x_{0}$ is constant point. If all $\beta_{i}$ 's are mutually different, $M^{n}$ is said to be of $L_{k}$-m-type. An $L_{k}$ - $m$-type hypersurface is said to be null if for at least one $i(1 \leq i \leq m)$ we have $\beta_{i}=0$.

Now, we see two examples of $L_{k}$-biconservative timelike hypersurfaces in $E_{1}^{4}$, for $k=0,1,2$.

Example 2.6. Assume that $\mathcal{D}_{1}(r)$ be the product $S_{1}^{2}(r) \times R \subset E_{1}^{4}$ where $r>0$. It has another representation as

$$
\mathcal{D}_{1}(r)=\left\{\left(y_{1}, \ldots, y_{4}\right) \in E_{1}^{4} \mid-y_{1}^{2}+y_{2}^{2}+y_{3}^{2}=r^{2}\right\},
$$

having the spacelike vector field $\mathbf{n}(y)=-\frac{1}{r}\left(y_{1}, y_{2}, y_{3}, 0\right)$ as the Gauss map. Clearly, it has two distinct principal curvatures $\kappa_{1}=\kappa_{2}=\frac{1}{r}, \kappa_{3}=0$, and the constant higher order mean curvatures $H_{1}=\frac{2}{3} r^{-1}, H_{2}=\frac{1}{3} r^{-2}$ and $H_{3}=0$.

Example 2.7. Assume that $\mathcal{D}_{2}(r)$ be the product $S_{1}^{1}(r) \times E^{2} \subset E_{1}^{4}$ where $r>0$. It has another representation as

$$
\mathcal{D}_{2}(r)=\left\{\left(y_{1}, \ldots, y_{4}\right) \in E_{1}^{4} \mid-y_{1}^{2}+y_{2}^{2}=r^{2}\right\},
$$

having the spacelike vector field $\mathbf{n}(y)=-\frac{1}{r}\left(y_{1}, y_{2}, 0,0,0\right)$ as the Gauss map. Clearly, it has two distinct principal curvatures $\kappa_{1}=\frac{1}{r}, \kappa_{2}=\kappa_{3}=0$, and the constant higher order mean curvatures $H_{1}=\frac{1}{4 r}$, and $H_{2}=H_{3}=0$.

Example 2.8. Let $\mathcal{D}_{3}(r)$ be the product $E_{1}^{2} \times S^{1}(r) \subset E_{1}^{4}$ where $r>0$. It can be represented as

$$
\mathcal{D}_{3}(r)=\left\{\left(y_{1}, \ldots, y_{4}\right) \in E_{1}^{4} \mid y_{4}^{2}+y_{5}^{2}=r^{2}\right\},
$$

with the Gauss map $\mathbf{n}(y)=-\frac{1}{r}\left(0,0,0, y_{4}, y_{5}\right)$. it has two distinct principal curvatures $\kappa_{1}=\kappa_{2}=0, \kappa_{3}=\frac{1}{r}$, and the constant higher order mean curvatures $H_{1}=\frac{1}{4 r}$, and $H_{k}=0$ for $k=2,3$. 
Example 2.9. Consider the pseudo-sphere

$$
S_{1}^{3}(r)=\left\{\left(y_{1}, \ldots, y_{4}\right) \in E_{1}^{4} \mid-y_{1}^{2}+y_{2}^{2}+y_{3}^{2}+y_{4}^{2}=r^{2}\right\},
$$

(for $r>0$ ) with the Gauss map $\mathbf{n}(y)=-\frac{1}{r}\left(y_{1}, y_{2}, y_{3}, y_{4}\right)$. It has three principal curvatures $\kappa_{1}=\kappa_{2}=\kappa_{3}=\frac{1}{r}$ and constant higher order mean curvatures $H_{k}=\frac{1}{r^{k}}$, for $k=1,2,3$.

\section{Results}

In this section, we give four theorems on the $L_{k}$-biconservative connected orientable timelike hypersurface in $E_{1}^{4}$ with constant ordinary mean curvature. Theorem 3.1 is appropriated to the case that the shape operator on hypersurface is diagonalizable. Theorems 3.2,3.3 and 3.4 are related to the cases that the shape operator on hypersurface is of type $I I, I I I$ and $I V$, respectively.

Theorem 3.1. Let $x: M_{1}^{3} \rightarrow E_{1}^{4}$ be a $L_{k}$-biconservative timelike hypersurface in the Minkowski 4-space, having diagonalizable shape operator (i.e of type $I$ ) with constant $k$ th mean curvature and exactly two distinct principal curvatures (for $k$ a nonnegative integer number less than 3). Then, it has constant $(k+1)$ th mean curvature.

Proof. By assumption, $M_{1}^{3}$ has two distinct principal curvatures $\lambda$ and $\mu$ of multiplicities 2 and 1 , respectively. Defining the open subset $U$ of $M_{1}^{3}$ as $U:=\left\{p \in M_{1}^{3}: \nabla H_{k+1}^{2}(p) \neq 0\right\}$, we prove that $U$ is empty. Assuming $U \neq \emptyset$, we consider $\left\{e_{1}, e_{2}, e_{3}\right\}$ as a local orthonormal frame of principal directions of $S$ on $\mathcal{U}$ such that $S e_{i}=\lambda_{i} e_{i}$ for $i=1,2,3$. By assumption, we have

$$
\lambda_{1}=\lambda_{2}=\lambda, \quad \lambda_{3}=\mu .
$$

Therefore, we obtain

$$
\mu_{1,2}=\mu_{2,2}=\lambda \mu, \quad \mu_{3,2}=\lambda^{2}, \quad 3 H_{2}=\lambda^{2}+2 \lambda \mu .
$$

In the case $k=1$, by condition $(2.9)(i i)$, we have

$$
P_{2}\left(\nabla H_{2}\right)=\frac{9}{2} H_{2} \nabla H_{2} .
$$

Then, using the polar decomposition 


$$
\nabla H_{2}=\sum_{i=1}^{3} \epsilon_{i}<\nabla H_{2}, e_{i}>e_{i}
$$

we see that (3.2) is equivalent to

$$
\epsilon_{i}<\nabla H_{2}, e_{i}>\left(\mu_{i, 2}-\frac{9}{2} H_{2}\right)=0
$$

on $\mathcal{U}$ for $i=1,2,3$. Hence, for every $i$ such that $<\nabla H_{2}, e_{i}>\neq 0$ on $\mathcal{U}$ we get

$$
\mu_{i, 2}=\frac{9}{2} H_{2} .
$$

By definition, we have $\nabla H_{2} \neq 0$ on $U$, which gives one or both of the following states.

State 1. $<\nabla H_{2}, e_{i}>\neq 0$, for $i=1$ or $i=2$. By equalities (3.1) and (3.4), we obtain

$$
\lambda \mu=\frac{9}{2}\left(\frac{2}{3} \lambda \mu+\frac{1}{3} \lambda^{2}\right)
$$

which gives

$$
\lambda\left(6 H-\frac{5}{2} \lambda\right)=0 .
$$

If $\lambda=0$ then $H_{2}=0$. Otherwise, we get $\lambda=\frac{12}{5} H, \mu=-\frac{9}{5} H$ and $H_{2}=-\frac{72}{25} H^{2}$.

State 2. $<\nabla H_{2}, e_{3}>\neq 0$. By equalities (3.1) and (3.4), we obtain

$$
\lambda^{2}=\frac{9}{2}\left(\frac{2}{3} \lambda \mu+\frac{1}{3} \lambda^{2}\right)
$$

which gives

$$
\lambda\left(9 H-\frac{11}{2} \lambda\right)=0 .
$$

If $\lambda=0$ then $H_{2}=0$. Otherwise, we have $\lambda=\frac{18}{11} H, \mu=-\frac{3}{11} H$ and $H_{2}=\frac{216}{121} H^{2}$.

Therefore, $\mathrm{H}_{2}$ is constant.

In the case $k=0$, the main claim is proven in $[3,4]$. In the case $k=2$, from condition (2.10)(ii) we get $e_{i}\left(H_{3}^{2}\right)=0$ for $i=1,2,3$, which means that there is nothing to prove. 
Theorem 3.2. Let $k$ be a nonnegative integer number less than 3 and $x: M_{1}^{3} \rightarrow E_{1}^{4}$ be an $L_{k}$-biconservative connected orientable timelike hypersurface with shape operator of type II which has exactly two distinct principal curvatures and constant $k$ th mean curvature, then it's $(k+1)$ th mean curvature is constant.

Proof. Suppose that, $\mathrm{H}_{2}$ is non-constant. Considering the open subset $\mathcal{U}=\left\{p \in M: \nabla H_{2}^{2}(p) \neq 0\right\}$, we try to show $\mathcal{U}=\emptyset$. By the assumption, with respect to a suitable (local) orthonormal tangent frame $\left\{e_{1}, e_{2}, e_{3}\right\}$ on $M$, the shape operator $S$ has the matrix form $\tilde{B}_{2}$, such that $S e_{1}=$ $\left(\kappa+\frac{1}{2}\right) e_{1}-\frac{1}{2} e_{2}, S e_{2}=\frac{1}{2} e_{1}+\left(\kappa-\frac{1}{2}\right) e_{2}, S e_{3}=\lambda e_{3}$ and then, we have $P_{2} e_{1}=\left(\kappa-\frac{1}{2}\right) \lambda e_{1}+\frac{1}{2} \lambda e_{2}, P_{2} e_{2}=-\frac{1}{2} \lambda e_{1}+\left(\kappa+\frac{1}{2}\right) \lambda e_{2}$ and $P_{2} e_{3}=\kappa^{2} e_{3}$.

When $k=0$, the result is derived from [3, 4]. In the case $k=1$, by condition $(2.9)(i i)$, using the polar decomposition $\nabla H_{2}=\sum_{i=1}^{3} \epsilon_{i} e_{i}\left(H_{2}\right) e_{i}$, we get

$$
\begin{aligned}
& \text { (i) } \epsilon_{1} e_{1}\left(H_{2}\right)\left[\left(\kappa-\frac{1}{2}\right) \lambda-\frac{9}{2} H_{2}\right]=\epsilon_{2} e_{2}\left(H_{2}\right) \frac{\lambda}{2} \\
& \text { (ii) } \epsilon_{2} e_{2}\left(H_{2}\right)\left[\left(\kappa+\frac{1}{2}\right) \lambda-\frac{9}{2} H_{2}\right]=-\epsilon_{1} e_{1}\left(H_{2}\right) \frac{\lambda}{2}, \\
& \text { (iii) } \epsilon_{3} e_{3}\left(H_{2}\right)\left(\kappa^{2}-\frac{9}{2} H_{2}\right)=0 .
\end{aligned}
$$

Now, we prove some simple claims.

Claim 1: $e_{1}\left(H_{2}\right)=e_{2}\left(H_{2}\right)=e_{3}\left(H_{2}\right)=0$.

If $e_{1}\left(H_{2}\right) \neq 0$, then by dividing both sides of equalities $(3.7)(i, i i)$ by $\epsilon_{1} e_{1}\left(H_{2}\right)$ we get

$$
\begin{aligned}
& \text { (i) }\left(\kappa-\frac{1}{2}\right) \lambda-\frac{9}{2} H_{2}=\frac{\epsilon_{2} e_{2}\left(H_{2}\right)}{\epsilon_{1} e_{1}\left(H_{2}\right)} \frac{\lambda}{2}, \\
& \text { (ii) } \frac{\epsilon_{2} e_{2}\left(H_{2}\right)}{\epsilon_{1} e_{1}\left(H_{2}\right)}\left[\left(\kappa+\frac{1}{2}\right) \lambda-\frac{9}{2} H_{2}\right]=-\frac{\lambda}{2},
\end{aligned}
$$

which, by substituting $(i)$ in $(i i)$, gives $\frac{\lambda}{2}(1+u)^{2}=0$, where $u:=\frac{\epsilon_{2} e_{2}\left(H_{2}\right)}{\epsilon_{1} e_{1}\left(H_{2}\right)}$. Then $\lambda=0$ or $u=-1$. If $\lambda=0$, then we get $H_{2}=0$ from $(3.8)(i)$. Also, by assumption $\lambda \neq 0$ we get $u=-1$ which gives $\kappa \lambda=\frac{9}{2} H_{2}$, then $\kappa(3 \kappa+4 \lambda)=0$ and finally $\kappa=-\frac{4}{3} \lambda$ (since $\kappa=0$ gives $H_{2}=0$ again). Hence, we have $H_{2}=\frac{2}{9} \kappa \lambda=-\frac{8}{27} \lambda^{2}$ and $H_{1}=-\frac{5}{9} \lambda$, and since $H_{1}$ is assumed to be constant, then $\lambda=-\frac{9}{5} H_{1}$ and $H_{2}=-\frac{24}{25} H_{1}^{2}$ have to be constant and we have $e_{1}\left(H_{2}\right)=0$, which is a contradiction. Therefore, the first claim is proved.

The second part of Claim 1 is $e_{2}\left(H_{2}\right)=0$. It can be proven by a similar manner. If $e_{2}\left(H_{2}\right) \neq 0$, then by dividing both sides of equalities $(3.7)(i, i i)$ by $\epsilon_{2} e_{2}\left(H_{2}\right)$ we get 
(i) $\frac{\epsilon_{1} e_{1}\left(H_{2}\right)}{\epsilon_{2} e_{2}\left(H_{2}\right)}\left[\left(\kappa-\frac{1}{2}\right) \lambda-\frac{9}{2} H_{2}\right]=\frac{\lambda}{2}$

(ii) $\left(\kappa+\frac{1}{2}\right) \lambda-\frac{9}{2} H_{2}=-\frac{\epsilon_{1} e_{1}\left(H_{2}\right)}{\epsilon_{2} e_{2}\left(H_{2}\right)} \frac{\lambda}{2}$,

which, by substituting $(i i)$ in $(i)$, gives $\frac{\lambda}{2}(1+v)^{2}=0$, where $v:=\frac{\epsilon_{1} e_{1}\left(H_{2}\right)}{\epsilon_{2} e_{2}\left(H_{2}\right)}$. Then $\lambda=0$ or $v=-1$. If $\lambda=0$, then we get $H_{2}=0$ from (3.9)(ii). Also, by assumption $\lambda \neq 0$ we get $v=-1$ which gives $\kappa \lambda=\frac{9}{2} H_{2}$, then $\kappa(3 \kappa+4 \lambda)=0$ and finally $\kappa=-\frac{4}{3} \lambda$ (since $\kappa=0$ gives $H_{2}=0$ again). Hence, we have $H_{2}=\frac{2}{9} \kappa \lambda=-\frac{8}{27} \lambda^{2}$ and $H_{1}=-\frac{5}{9} \lambda$, and since $H_{1}$ is assumed to be constant, then $\lambda=-\frac{9}{5} H_{1}$ and $H_{2}=-\frac{24}{25} H_{1}^{2}$ have to be constant and we have $e_{2}\left(H_{2}\right)=0$, which is a contradiction. Therefore, the second part of Claim 1 is proved.

Now, if $e_{3}\left(H_{2}\right) \neq 0$, then by $(3.7)($ iii $)$ we get $\kappa^{2}=\frac{9}{2} H_{2}$, then $\kappa(\kappa+6 \lambda)=$ 0 , which gives $\kappa=0$ or $\kappa=-6 \lambda$. If $\kappa=0$, then $H_{2}=0$, and if $\kappa=-6 \lambda$ then since $H_{1}=-\frac{11}{3} \lambda$ is assumed to be constant, we get that $H_{2}$ is constant and then $e_{3}\left(H_{2}\right)=0$. Which is a contradiction, so we have $e_{3}\left(H_{2}\right)=0$.

In the case $k=2$, from condition $(2.10)$ (ii) we get $e_{i}\left(H_{3}^{2}\right)=0$ for $i=1,2,3$, which means that there is nothing to prove.

Theorem 3.3. Let $k$ be a nonnegative integer number less than 3 and $x: M_{1}^{3} \rightarrow E_{1}^{4}$ be an $L_{k}$-biconservative connected orientable timelike hypersurface with shape operator of type III which has constant kth mean curvature, then it's $(k+1)$ th mean curvature is constant.

Proof. When $k=0$, the result is derived from [3, 4]. In the case $k=1$, suppose that, $H_{2}$ is non-constant. Considering the open subset $\mathcal{U}=\left\{p \in M: \nabla H_{2}^{2}(p) \neq 0\right\}$, we try to show $\mathcal{U}=\emptyset$. By the assumption, with respect to a suitable (local) orthonormal tangent frame $\left\{e_{1}, e_{2}, e_{3}\right\}$ on $M$, the shape operator $S$ has the matrix form $\tilde{B}_{3}$, such that $S e_{1}=$ $\kappa e_{1}+\frac{\sqrt{2}}{2} e_{3}, S e_{2}=\kappa e_{2}-\frac{\sqrt{2}}{2} e_{3}, S e_{3}=-\frac{\sqrt{2}}{2} e_{1}-\frac{\sqrt{2}}{2} e_{2}+\kappa e_{3}$ and then, we have $P_{2} e_{1}=\left(\kappa^{2}-\frac{1}{2}\right) e_{1}-\frac{1}{2} e_{2}-\frac{\sqrt{2}}{2} \kappa e_{3}, P_{2} e_{2}=\frac{1}{2} e_{1}+\left(\kappa^{2}+\frac{1}{2}\right) e_{2}+\frac{\sqrt{2}}{2} \kappa e_{3}$ and $P_{2} e_{3}=\frac{\sqrt{2}}{2} \kappa e_{1}+\frac{\sqrt{2}}{2} \kappa e_{2}+\kappa^{2} e_{3}$.

Using the polar decomposition $\nabla H_{2}=\sum_{i=1}^{3} \epsilon_{i} e_{i}\left(H_{2}\right) e_{i}$, from condition (2.9)(ii) we get

(i) $\epsilon_{1} e_{1}\left(H_{2}\right)\left[\left(\kappa^{2}-\frac{1}{2}\right)-\frac{9}{2} H_{2}\right]+\frac{1}{2} \epsilon_{2} e_{2}\left(H_{2}\right)+\frac{\sqrt{2}}{2} \epsilon_{3} e_{3}\left(H_{2}\right) \kappa=0$,

$(3.10)(i i) \frac{-1}{2} \epsilon_{1} e_{1}\left(H_{2}\right)+\epsilon_{2} e_{2}\left(H_{2}\right)\left[\left(\kappa^{2}+\frac{1}{2}\right)-\frac{9}{2} H_{2}\right]+\frac{\sqrt{2}}{2} \epsilon_{3} e_{3}\left(H_{2}\right) \kappa=0$,

(iii) $\epsilon_{1} e_{1}\left(H_{2}\right) \frac{-\sqrt{2}}{2} \kappa+\epsilon_{2} e_{2}\left(H_{2}\right) \frac{\sqrt{2}}{2} \kappa+\epsilon_{3} e_{3}\left(H_{2}\right)\left(\kappa^{2}-\frac{9}{2} H_{2}\right)=0$, 
Now, we prove some simple claims.

Claim: $e_{1}\left(H_{2}\right)=e_{2}\left(H_{2}\right)=e_{3}\left(H_{2}\right)=0$.

If $e_{1}\left(H_{2}\right) \neq 0$, then by dividing both sides of equalities $(3.10)(i, i i, i i i)$ by $\epsilon_{1} e_{1}\left(H_{2}\right)$, and using the identity $H_{2}=\kappa^{2}$ in type $I I I$, we get

$$
\begin{aligned}
& \text { (i) }-\frac{1}{2}-\frac{7}{2} \kappa^{2}+\frac{1}{2} u_{1}+\frac{\sqrt{2}}{2} u_{2} \kappa=0, \\
& \text { (ii) } \frac{-1}{2}+u_{1}\left(\frac{1}{2}-\frac{7}{2} \kappa^{2}\right)+\frac{\sqrt{2}}{2} u_{2} \kappa=0, \\
& \text { (iii) } \left.\frac{-\sqrt{2}}{2} \kappa+\frac{\sqrt{2}}{2} u_{1} \kappa-\frac{7}{2} \kappa^{2}\right) u_{2}=0,
\end{aligned}
$$

where $u_{1}:=\frac{\epsilon_{2} e_{2}\left(H_{2}\right)}{\epsilon_{1} e_{1}\left(H_{2}\right)}$ and $u_{2}:=\frac{\epsilon_{3} e_{3}\left(H_{2}\right)}{\epsilon_{1} e_{1}\left(H_{2}\right)}$, which, by comparing $(i)$ and $(i i)$, gives $\kappa^{2}\left(u_{1}-1\right)=0$. If $\kappa=0$, then $H_{2}=0$. Assuming $\kappa \neq 0$, we get $u_{1}=1$, which, using (3.11)(iii), gives $u_{2}=0$. Substituting $u_{1}=1$ and $u_{2}=0$ in $(3.11)(i)$, we obtain again $\kappa=0$, which is a contradiction. Hence $e_{1}\left(H_{2}\right) \equiv 0$.

Therefore, using the result $e_{1}\left(H_{2}\right) \equiv 0$, the system of equations (3.10) gives

$$
\begin{aligned}
& \text { (i) } \frac{1}{2} \epsilon_{2} e_{2}\left(H_{2}\right)+\frac{\sqrt{2}}{2} \epsilon_{3} e_{3}\left(H_{2}\right) \kappa=0, \\
& \text { (ii) } \epsilon_{2} e_{2}\left(H_{2}\right)\left(\frac{1}{2}-\frac{7}{2} \kappa^{2}\right)+\frac{\sqrt{2}}{2} \epsilon_{3} e_{3}\left(H_{2}\right) \kappa=0, \\
& \text { (iii) } \epsilon_{2} e_{2}\left(H_{2}\right) \frac{\sqrt{2}}{2} \kappa-\epsilon_{3} e_{3}\left(H_{2}\right) \frac{7}{2} \kappa^{2}=0 .
\end{aligned}
$$

Comparing parts $(i),(i i)$ and $(i i i)$ of (3.12), we get $\kappa e_{2}\left(H_{2}\right)=0$ and $\kappa e_{3}\left(H_{2}\right)=0$, hence, using $(i)$, gives $e_{2}\left(H_{2}\right)=0$. Then, the second claim (i.e. $\left.e_{2}\left(H_{2}\right)=0\right)$ is proved.

Now, using the results $e_{1}\left(H_{2}\right)=e_{2}\left(H_{2}\right)=0$, we get $\kappa e_{3}\left(H_{2}\right)=0$, which, using $H_{2}=\kappa^{2}$, implies $\kappa_{3}\left(\kappa^{2}\right)=0$ and then $e_{3}\left(\kappa^{3}\right)=0$, and finally $e_{3}\left(H_{2}\right)=0$.

In the case $k=2$, from condition (2.10)(ii) we get $e_{i}\left(H_{3}^{2}\right)=0$ for $i=1,2,3$, which means that there is nothing to prove.

Theorem 3.4. Let $k$ be a nonnegative integer number less than 3 and $x: M_{1}^{3} \rightarrow E_{1}^{4}$ be an $L_{k}$-biconservative connected orientable timelike hypersurface with shape operator of type $I V$ which has constant $k$ th mean curvature and a constant real principal curvature. Then, its 2 nd and $3 r d$ mean curvatures are constant.

Proof. When $k=0$, the result is derived from [3, 4]. In the case $k=1$, suppose that, $H_{2}$ is non-constant. Considering the open subset $\mathcal{U}=\left\{p \in M: \nabla H_{2}^{2}(p) \neq 0\right\}$, we try to show $\mathcal{U}=\emptyset$. By the assumption 
$M_{1}^{3}$ has three distinct principal curvature, then, with respect to a suitable (local) orthonormal tangent frame $\left\{e_{1}, e_{2}, e_{3}\right\}$ on $M$, the shape operator $S$ has the matrix form $B_{4}$, such that $S e_{1}=\kappa e_{1}-\lambda e_{2}, S e_{2}=\lambda e_{1}+\kappa e_{2}$, $S e_{3}=\eta e_{3}$ and then, we have $P_{2} e_{1}=\kappa \eta e_{1}+\lambda \eta e_{2}, P_{2} e_{2}=-\lambda \eta e_{1}+\kappa \eta e_{2}$ and $P_{2} e_{3}=\left(\kappa^{2}+\lambda^{2}\right) e_{3}$.

Using the polar decomposition $\nabla H_{2}=\sum_{i=1}^{3} \epsilon_{i} e_{i}\left(H_{2}\right) e_{i}$, from condition (2.9)(ii) we get

$$
\begin{aligned}
& \text { (i) } \epsilon_{1} e_{1}\left(H_{2}\right)\left(\kappa \eta-\frac{9}{2} H_{2}\right)=\epsilon_{2} e_{2}\left(H_{2}\right) \lambda \eta \\
& \text { (ii) } \epsilon_{2} e_{2}\left(H_{2}\right)\left(\kappa \eta-\frac{9}{2} H_{2}\right)=-\epsilon_{1} e_{1}\left(H_{2}\right) \lambda \eta \\
& \text { (iii) } \epsilon_{3} e_{3}\left(H_{2}\right)\left(\kappa^{2}+\lambda^{2}-\frac{9}{2} H_{2}\right)=0 .
\end{aligned}
$$

Now, we prove three simple claims.

Claim 1: $e_{1}\left(H_{2}\right)=e_{2}\left(H_{2}\right)=0$.

If $e_{1}\left(H_{2}\right) \neq 0$, then by dividing both sides of equalities $(3.13)(i, i i)$ by $\epsilon_{1} e_{1}\left(H_{2}\right)$ we get

$$
\begin{aligned}
& \text { (i) } \kappa \eta-\frac{9}{2} H_{2}=\frac{\epsilon_{2} e_{2}\left(H_{2}\right)}{\epsilon_{1} e_{1}\left(H_{2}\right)} \lambda \eta, \\
& \text { (ii) } \frac{\epsilon_{2} e_{2}\left(H_{2}\right)}{\epsilon_{1} e_{1}\left(H_{2}\right)}\left(\kappa \eta-\frac{9}{2} H_{2}\right)=-\lambda \eta,
\end{aligned}
$$

which, by substituting $(i)$ in $(i i)$, gives $\lambda \eta\left(1+\left(\frac{\epsilon_{2} e_{2}\left(H_{2}\right)}{\epsilon_{1} e_{1}\left(H_{2}\right)}\right)^{2}\right)=0$, then $\lambda \eta=0$. Since by assumption $\lambda \neq 0$, we get $\eta=0$. So, by $(3.14(i))$, we have $H_{2}=0$.

Similarly, if $e_{2}\left(H_{2}\right) \neq 0$, then by dividing both sides of $(3.13(i, i i))$ by $\epsilon_{2} e_{2}\left(H_{2}\right)$ we get

$$
\begin{aligned}
& \text { (i) } \frac{\epsilon_{1} e_{1}\left(H_{2}\right)}{\epsilon_{2} e_{2}\left(H_{2}\right)}\left(\kappa \eta-\frac{9}{2} H_{2}\right)=\lambda \eta \\
& \text { (ii) } \kappa \eta-\frac{9}{2} H_{2}=-\frac{\epsilon_{1} e_{1}\left(H_{2}\right)}{\epsilon_{2} e_{2}\left(H_{2}\right)} \lambda \eta
\end{aligned}
$$

which, by substituting $(i)$ in $(i i)$, gives $\lambda \eta\left(1+\left(\frac{\epsilon_{1} e_{1}\left(H_{2}\right)}{\epsilon_{2} e_{2}\left(H_{2}\right)}\right)^{2}\right)=0$, then $\lambda \eta=0$. Since by assumption $\lambda \neq 0$, we get $\eta=0$. So, by $(3.15(i i))$, we have $H_{2}=0$.

Claim 2: $e_{3}\left(H_{2}\right)=0$.

If $e_{3}\left(H_{2}\right) \neq 0$, then from equality $(3.13(i i i))$ we have $\kappa^{2}+\lambda^{2}=\frac{9}{2} H_{2}$, which gives $\kappa^{2}+\lambda^{2}=-6 \kappa \eta$, where $\eta=3 H_{1}-2 \kappa$ and $\eta$ and $H_{1}$ are assumed to be constant on $U$. So, $\kappa$ is also constant on $U$, and then, we obtain $H_{2}=\frac{-4}{3} \kappa \eta=\frac{8}{3} \kappa^{2}-4 H_{1} \kappa$ and $H_{3}=-6 \kappa \eta^{2}=-6 \kappa\left(3 H_{1}-2 \kappa\right)^{2}$. are constant on $U$.

In the case $k=2$, from condition (2.10)(ii) we get $e_{i}\left(H_{3}^{2}\right)=0$ for $i=1,2,3$, which means that there is nothing to prove. 


\section{References}

[1] K. Akutagawa, and S. Maeta, "Biharmonic properly immersed submanifolds in Euclidean spaces", Geometriae Dedicata, vol. 164, pp. 351-355, 2013.

[2] L. J. Alias, and N Gürbüz, "An extension of Takahashi theorem for the linearized operators of the higher order mean curvatures", Geometriae Dedicata, vol. 121, pp. 113-127, 2006.

[3] A. Arvanitoyeorgos, F. Defever, G. Kaimakamis, and B. J. Papantoniou, "Biharmonic Lorentz hypersurfaces in $\mathrm{E}^{4}{ }_{1}$ ", Pacific Journal of Mathematics, vol. 229, no. 2, pp. 293-306, 2007.

[4] A. Arvanitoyeorgos, F. Defever, and G. Kaimakamis, "Hypersurfaces in $\mathrm{E}^{4} \mathrm{~s}$ with proper mean curvature vector", Journal of the Mathematical Society of Japan, vol. 59, no. 3, pp. 797-809, 2007.

[5] B. Y. Chen, Total Mean Curvature and Submanifolds of Finite Type, Singapure: World Scientific Publishing, 2014.

[6] B. Y. Chen, "Some open problems and conjetures on submanifolds of finite type", Soochow Journal of Mathematics, vol. 17, pp. 169-188, 1991.

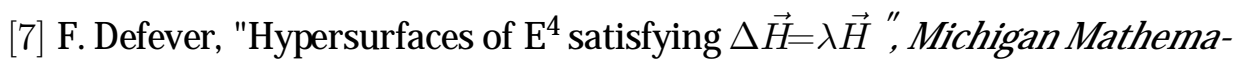
tical Journal, vol. 44, pp. 355-363, 1997.

[8] F. Defever, "Hypersurfaces of $\mathrm{E}^{4}$ with harmonic mean curvature vector", Mathematische Nachrichten, vol. 196, pp.61-69, 1998.

[9] I. Dimitrić, "Submanifolds of En with harmonic mean curvature vector", Bulletin of Institute of Mathematics, Academia sinica. New series, vol. 20, pp. 53-65, 1992.

[10] T. Hasanis, and T. Vlachos, "Hypersurfaces in $\mathrm{E}^{4}$ with harmonic mean curvature vector field", Mathematische Nachrichten, vol. 172, pp. 145-169, 1995.

[11] S. M. B. Kashani, "On some L1-finite type (hyper)surfaces in $\mathrm{R}^{\mathrm{n}+1}$ ", Bulletin of the Korean Mathematical Society, vol. 46, no. 1, pp. 35-43, 2009. 
[12] P. Lucas, and H. F. Ramirez-Ospina, "Hypersurfaces in the LorentzMinkowski space satisfying $L k \psi=A \psi+b$ ", Geometriae Dedicata, vol. 153, pp. 151-175, 2011.

[13] M. A. Magid, "Lorentzian isoparametric hypersurfaces”, Pacific Journal of Mathematics, vol. 118, no. 1, pp. 165-197, 1985.

[14] F. Pashaie, and A. Mohammadpouri, "Lk-biharmonic spacelike hypersurfaces in Minkowski 4-space $\mathrm{E}^{4}{ }_{1}$ ", Sahand Communications in Mathematical Analysis, vol. 5, no. 1, pp. 21-30, 2017.

[15] B. O’Neill, Semi-Riemannian Geometry with Applications to Relativity. New York: Academic Press, 1983.

[16] F. Pashaie, and S. M. B. Kashani, "Spacelike hypersurfaces in Riemannian or Lorentzian space forms satisfying $\mathrm{Lkx}=\mathrm{Ax}+\mathrm{b}$ ", Bulletin of the Iranian Mathematical Society, vol. 39, no. 1, pp. 195-213, 2013.

[17] F. Pashaie, and S. M. B. Kashani, "Timelike hypersurfaces in the Lorentzian standard space forms satisfying $\mathrm{LkX}=\mathrm{Ax}+\mathrm{b}$ ", Mediterranean Journal of Mathematics, vol. 11, no. 2, pp. 755-773, 2014.

[18] A. Z. Petrov, Einstein Spaces. New York: Pergamon Press, 1969.

[19] R. C. Reilly, "Variational properties of functions of the mean curvatures for hypersurfaces in space forms", Journal of Differential Geometry, vol. 8, no. 3, pp. 465-477, 1973.

\author{
Firooz Pashaie \\ Dept. of Math, \\ Faculty of Basic Sciences, \\ University of Maragheh, \\ P. O. Box 55181-83111, Maragheh, \\ Iran \\ e-mail: f_pashaie@maragheh.ac.ir
}

